Canadian

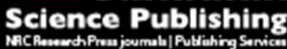

Applied Physiology, Nutrition, and Metabolism Physiologie appliquée, nutrition et métabolisme

\title{
The Cellular Stress Response of Rat Skeletal Muscle Following Lengthening Contractions
}

\begin{tabular}{|r|l|}
\hline Journal: & Applied Physiology, Nutrition, and Metabolism \\
\hline Manuscript ID & apnm-2016-0556.R2 \\
\hline Manuscript Type: & Article \\
\hline Date Submitted by the Author: & 23-Jan-2017 \\
\hline Complete List of Authors: & $\begin{array}{l}\text { Pollock-Tahiri, Evan; University of Toronto } \\
\text { Locke, Marius; University of Toronto, }\end{array}$ \\
\hline Keyword: & $\begin{array}{l}\text { skeletal muscle damage, heat shock proteins, heat shock transcription } \\
\text { factor, lengthening muscle contraction }\end{array}$ \\
\hline \multicolumn{2}{|l}{} \\
\hline
\end{tabular}

\section{SCHOLARONE \\ Manuscripts}


The Cellular Stress Response of Rat Skeletal Muscle Following Lengthening Contractions

\author{
Evan Pollock-Tahiri and Marius Locke, \\ Faculty of Kinesiology and Physical Education \\ University of Toronto, \\ 55 Harbord Street \\ Toronto, Ontario, \\ M5S 2W6 \\ Canada
}

Running Title: Lengthening Contractions and Heat Shock Proteins

Correspondence:

Marius Locke, PhD.

Faculty of Kinesiology and Physical Education

University of Toronto,

55 Harbord Street

Toronto, Ontario,

M5S 2W6

Canada

Telephone: 416 978-7055

Fax:416 978-6978

E-mail: marius.locke@utoronto.ca 


\section{Abstract}

The cellular stress response (CSR) of the rat tibialis anterior (TA) muscle was investigated following 20, 40 or 60 lengthening contractions (LCs) using an in-vivo model of electrical stimulation. Muscles were removed at $0,1,3$, or 24 hours after LCs and assessed for HSF activation, HSP content and/or morphological evidence of muscle fibre damage. When compared to the first muscle contraction, peak muscle torque was reduced by $26 \%(\mathrm{p}<0.05)$ after $20 \mathrm{LCs}$ and further reduced to $56 \%$ and $60 \%$ $(\mathrm{p}<0.001)$ after 40 and $60 \mathrm{LCs}$, respectively. Following $60 \mathrm{LCs}$, HSF activation was detected at 0,1 and 3 hours but was undetectable at 24 hours. Hsp72 content was elevated at 24 hours after 20 LCs (2.34 \pm 0.37 fold, $\mathrm{p}<0.05), 40$ LCs $(3.02 \pm 0.31$ fold, $\mathrm{p}<0.01)$ and 60 LCs $(3.37 \pm 0.21$ fold, $\mathrm{p}<0.001)$. Hsp25 content increased after $40(2.36 \pm 0.24$ fold, $\mathrm{p}<0.01)$ and 60 LCs $(2.80 \pm 0.37$ fold, $\mathrm{p}<0.01)$. Morphological assessment of TA morphology revealed very few fibres were damaged following 20 LCs while multiple sets of LCs (40 and 60) caused greater amounts of fiber damage. Electron microscopy showed disrupted Z-lines and sarcomeres were detectable in some muscles fibres following 20 LCs but were more prevalent and severe in muscles subjected to 40 or 60 LCs. These results suggest LCs elevate HSP content by an HSF mediated mechanism (60 LC) and a single set of 20 LCs is capable of increasing muscle HSP content without causing significant muscle fibre damage.

Key Words: lengthening muscle contractions, heat shock proteins, muscle damage, heat shock transcription factor, tibialis anterior. 


\section{Introduction}

Lengthening muscle contractions (LCs; also known as eccentric contractions) occur when a muscle lengthens while generating force. LCs are a means of dissipating energy and performed daily, such as while walking downstairs. However, when performed in excess, LCs are known to cause muscle damage that is characterized by a number of alterations including an impaired muscle contractile function, as well as damage to cytoskeletal and sarcomere structures (McCully and Faulkner 1985; Proske and Morgan 2001). Since LCs are commonly used in resistance training and rehabilitation programs, it follows that a better understanding of the cellular response and adaptations to LCs may allow for better evidence based training, treatments and rehabilitation programs.

When LCs are performed in excess and result in muscle damage, the cellular stress response (CSR) is initiated and intramuscular heat shock protein (HSP) content is increased (Holwerda and Locke 2014). HSPs are molecular chaperones that assist in the maintenance of proteostasis during cellular stress; however, their exact role in mammalian skeletal muscle remains to be fully understood. To date, most studies demonstrating LC-induced HSPs have inflicted significant amounts of muscle damage either by using protocols with an excessive contraction number or prolonged downhill running Paulsen et al. 2007; Touchberry et al. 2012). In contrast, mild or moderate intensity protocols using LCs which are more suitable for exercise and rehabilitation regimens, have not adequately investigated the CSR. Recent work has shown that 100 LCs increased muscle Hsp72 and Hsp25 content and muscle damage in the rat TA muscle while 100 isokinetically matched shortening contractions did not elevate intramuscular HSP content (Holwerda and Locke 2014). This suggests that the skeletal muscle HSP response during exercise may be the result of LC induced damage to cytoskeletal and sarcomeric structures which may activate the CSR and require upregulation of HSPs.

Stress induction of HSPs is regulated by proteins known as heat shock transcription factors 
(HSFs; Pirkkala et al. 2001). In unstressed cells, HSFs exist as monomers in the cytoplasm bound to an inhibitory complex of HSPs. Following stress, the accumulation of denatured proteins is thought to result in the dissociation of HSPs from the HSFs allowing HSF trimerization, nuclear localization and binding to the heat shock element (HSE; Morimoto, 1998). In mammalian skeletal muscle subjected to heat shock, HSF activation (HSF-HSE binding) shows rapid (0-2 hrs) activation and inactivation kinetics (Locke and Tanguay 1996; Locke 2001). To date, despite the presence of HSPs suggesting an HSF mediated mechanism, no studies have investigated HSF activation following LCs.

HSPs have been shown to protect cells and tissues, including skeletal muscle and thus based on these protective benefits (Senf 2013), it would be important to determine if HSP content can be increased by specific exercises without causing extensive muscle damage and/or compromising muscle function. Thus, the purpose of this study was to determine if HSP content could be increased in the rat TA muscle following a physiologically relevant number of LCs. A secondary purpose was to investigate the mechanism(s) of any LC-induced HSP content by assessing HSF activation (HSF-HSE binding) shortly after a single bout of 60 LCs.

\section{Methods and Materials}

\section{Animals}

Male Sprague-Dawley rats $(\mathrm{N}=38 ; \sim 350-410 \mathrm{~g})$ obtained from Charles River Laboratories (Quebec, Canada) were housed in pairs, maintained on a constant 12-hour light/dark cycle, and provided food and water ad libitum. All procedures were approved by the Animal Care Committee at the University of Toronto and were in accordance with Guidelines for Canadian Council on Animal Care. All experiments were performed under general anesthesia (Isoflourane/oxygen gas mixture; 1L/min). Following LCs, animals were monitored during recovery from anesthesia and were 
euthanized at selected time points (immediately, one, three or 24 hours) after experiments.

\section{Lengthening contractions and muscle contractile torque}

Anesthetized rats were placed in the supine position on a whole-body immobilization platform (806D, Aurora Scientific Inc., Aurora, Canada) maintained at $\sim 37^{\circ} \mathrm{C}$ with its left hindlimb secured to a foot-pedal. During all experiments, rats were under continuous inhalation anesthesia (Isoflourane/oxygen; 1L/min) using a fitted mask connected to a continuous-flow anesthetic machine (Harvard Apparatus, St. Laurent, Quebec). The left knee was secured between two vertical stabilizing posts by carefully inserting a $25 \mathrm{G} \times 1.5$-inch needle through the soft tissue of the knee and locked into position. Two subcutaneous electrodes were inserted adjacent to the proximal portion of the TA, parallel to the longitudinal axis of the tibia. The left paw was secured to a foot pedal connected to a computer-controlled servomotor (301C, Aurora Scientific Inc., Aurora, Canada). To induce LCs, the TA was stimulated to tetanus using a bi-phase stimulator (701C Aurora Scientific Inc., Aurora, Canada) connected to a computer controlled hardware interface (604A, Aurora Scientific Inc., Aurora, Canada) that could be adjusted using Dynamic Muscle Control software (DMC; 610A, Aurora Scientific Inc., Aurora, Canada). Optimal stimulation parameters were defined as the minimum voltage $(\mathrm{V})$ and frequency $(\mathrm{Hz})$ necessary to reach tetanus. In general, stimulation parameters were between six and ten volts at $150 \mathrm{~Hz}$. Peak muscle torque $(\mathrm{g}-\mathrm{cm})$ produced during each LC was acquired through the servomotor and transferred through a digital controller (604A, Aurora Scientific Inc., Aurora, Canada) to a computer running Dynamic Muscle Analysis (DMA) software (611A, Aurora Scientific Inc., Aurora, Canada). Each LC consisted of an isometric component (lasting $0.2 \mathrm{sec}$ ) for the TA to reach tetanus, immediately followed by a lengthening component (lasting $0.3 \mathrm{sec}$ ) over a range of $38^{\circ}$, thereby producing a maximal LC with an angular velocity of $127^{\circ} / \mathrm{sec}$. Following each LC, the servomotor passively returned the ankle to the starting position. Contraction protocols were organized into one, two, or three sets of 20 LCs ( $n=8$ per group) with five minutes of rest between sets. For the purposes of 
this study, peak torque was defined as the highest force generated by the muscle. To determine changes in muscle function, peak torque $(\mathrm{g}-\mathrm{cm})$ was measured during the $1^{\text {st }}, 5^{\text {th }}, 10^{\text {th }}, 15^{\text {th }}$, and last $\left(20^{\text {th }}\right)$ LC of each set and expressed as a percentage of initial muscle torque (i.e.; a percentage of the torque produced during the first LC).

\section{Tissue collection}

Rats were euthanized by anesthetized exsanguinations at various time points $(0,1,3,24 \mathrm{hrs})$ after LCs and the TA muscle was excised from both limbs (stimulated and contra-lateral control). Muscles were weighed and sliced into proximal, mid-belly and distal sections using two single-edge razor blades. The mid-belly portion was embedded in OCT and frozen in isopentane pre-cooled in liquid nitrogen. Proximal and distal portions were immediately frozen in liquid nitrogen. All samples were stored at $-80^{\circ} \mathrm{C}$.

\section{Protein determination and Western blotting}

Frozen TA samples were homogenized in 15 volumes of $600 \mathrm{mM} \mathrm{NaCl}, 15 \mathrm{mM}$ Tris, $\mathrm{pH} 7.5$ using a mechanical tissue grinder (IKA Labortechnik, Germany) and briefly centrifuged ( $20 \mathrm{sec})$ to pellet non-homogenized pieces or connective tissue. The supernatant was removed and protein concentrations determined using the method described by Lowry et al (1951) with bovine serum albumin (BSA) as a standard. Samples containing $100 \mu \mathrm{g}$ of protein were loaded into a 5-15\% gradient gel and separated by one-dimensional sodium dodecyl sulfate polyacrylamide gel electrophoresis (SDS-PAGE) with modifications previously described (Locke 2001; Laemmli 1970). Separated proteins were transferred from the gel to nitrocellulose membranes $(0.22-\mu \mathrm{m}$ pore size, Bio-Rad, Mississauga, Canada) as previously described (Towbin et al. 1979) and modified to the Bio-Rad miniprotein II gel transfer system (Frier and Locke 2007). Following transfer, membranes were blocked in 5\% Blotto (5\% non-fat skim milk powder in Tris Buffer Saline (TBS: 500mM NaCl, 20mM Tris, pH 7.5) for one hour. Blocked membranes were washed twice in TTBS (TBS plus 0.05\% Tween-20) and 
incubated overnight at $4^{\circ} \mathrm{C}$ in a polyclonal antibody specific to Hsp25 (ADI-SPP-715, Enzo, USA) or Hsp72 (ADI-SPA-812, Enzo, USA) diluted 1:1000 in TTBS with 2\% Blotto. Membranes were washed twice in TTBS for 5 minutes then incubated for one hour at room temperature in a 1:1000 dilution with goat anti-rabbit $(\mathrm{H}+\mathrm{L})$ secondary antibody conjugated to Alkaline Phosphatase (Bio-Rad \#170-65-18, Mississauga, Canada). Protein bands were visualized by incubating membranes with an NBT/BCIP (5Bromo-4-Chloro-3-Indolyl phosphate p-toluidine salt, p-Nitro-Blue-tetrazolium chloride) solution in $100 \mathrm{mM} \mathrm{Na} \mathrm{CO}_{3}, 1 \mathrm{mM} \mathrm{MgCl}_{2}, \mathrm{pH} 9.8$ in low light for approximately 10 minutes. Membranes were photographed and band densities were quantified using ImageJ software. In the case of Hsp25, both band densities were summed to represent total Hsp25 content. Values were compared to their contralateral control muscle and HSP content represented as a fold-difference from the unstimulated contralateral muscle where 1.0 suggests no change. A separate group of rats $(\mathrm{CON} ; \mathrm{n}=5)$ that received no stimulation were used to determine basal Hsp72 and Hsp25 content in the TA.

\section{Protein determination and electrophoretic mobility shift assay}

Frozen samples of the TA were homogenized in 15 volumes of extraction buffer $(25 \%$ glycerol, 0.42M NaCl, 1.5mM $\mathrm{MgCl}_{2}$, 0.5M EDTA; $\mathrm{pH}$ 8.0, 20mM Hepes; $\mathrm{pH}$ 7.5, 0.5mN DTT, 0.5M PMSF) on ice using a mechanical tissue grinder (IKA Labortechnik, Germany). Tissue lysates were centrifuged at 14,000 RPM for 20 minutes. The supernatant was extracted and protein concentrations determined using the Bradford assay with BSA as a standard. Electrophoretic Mobility Shift Assay

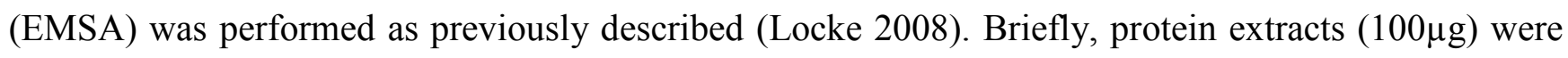
incubated with an ${ }^{32} \mathrm{P}$-labelled self-complimentary ideal HSE oligonucleotide (5'-CTA GAA GCT TCT AGA AGC TTC TAG-3') in binding buffer (10\% glycerol, 50mM NaCl, 1.0mM EDTA; pH 8.0, $20 \mathrm{mM}$ Tris; $\mathrm{pH} 8.0,1.0 \mathrm{mM}$ DTT, $0.3 \mathrm{mg} / \mathrm{ml} \mathrm{BSA}$ ) with $0.1 \mathrm{ng}$ (approximately $30-50,000 \mathrm{cpm}$ ) of ${ }^{32} \mathrm{P}-$ labelled oligonucleotide and $2 \mu \mathrm{g}$ poly dIdC (Pharmacia Fine Chemicals, Piscataway, NJ, USA) for 30 minutes at room temperature. Protein samples were loaded into a $4 \%$ polyacrylamide gel (5X running 
buffer; $\mathrm{pH} 8.5,30 \%$ acrylamide, $2 \%$ bis-acrylamide, $50 \%$ glycerol, $100 \mu 130 \%$ APS, $34 \mu 1$ TEMED) and electrophoresed at 200 volts for 2.5 hours. Immediately following electrophoresis, gels were transferred onto filter paper, dried using a BioRad Slab dryer (Model 433) for 45 minutes and exposed to high radiographic film (Amersham Hyperfilm ECL, GE Healthcare Limited) for 48 hours at $-80^{\circ} \mathrm{C}$.

\section{Muscle fiber morphology}

To assess fiber morphology, $10-15 \mu \mathrm{m}$ transverse sections of the TA mid-belly were cut in an American Optical cryostat at $-20^{\circ} \mathrm{C}$, air dried, stained with Hematoxylin and Eosin and viewed using a Zeiss Axioskop light microscope. Images were photographed using a Canon EOS Rebel T3i digital camera attached to the microscope using a Zarf Enterprise Microscope Adaptor (Spokane, Washington). Morphological characteristics and evidence of muscle fiber damage were evaluated using a qualitative method described for this study. Each muscle was examined at 10X magnification from one peripheral edge to the other for evidence of fiber damage. Fiber damage was identified by the presence of intramuscular or extracellular inflammatory cells, membrane disruptions, and necrosis (rounded/swollen fibers, eosinophilic fibers and degraded fibers which stain light). The severity of muscle damage was graded from mild to severe based on the extent of fiber damage and influx of inflammatory cells. Damage was considered mild when necrosis was localized to a single fiber within a single fascicle, and a low level of inflammatory infiltration. Conversely, the presence of multiple necrotic fibers within a single fascicle, or multiple effected fascicles, coupled with an abundance of inflammatory infiltrates in multiple muscle compartments (perimysium, epimysium and intramuscular) was considered severe damage.

\section{Electron Microscopy}

Portions of the mid belly of the rat TA were minced into 2-3 mm square pieces and placed in $3 \%$ glutaraldehyde in phosphate buffer $(\mathrm{pH} 7.4)$ for 2 days at $4^{\circ} \mathrm{C}$. Muscle pieces were washed in $0.1 \mathrm{M}$ Sorenson's phosphate buffer ( $3 \times 10 \mathrm{~min}$ each) and placed in $1 \% \mathrm{OsO}_{4}$ for one hour. After another 3 
washes, muscle pieces were dehydrated using an ethanol series (50\% $10 \mathrm{~min}, 70 \% 10 \mathrm{~min}, 80 \% 15$ $\min , 90 \% 2 \times 10 \mathrm{~min}, 100 \% 2 \times 10 \mathrm{~min})$. Muscle pieces were placed in an ethanol: Spurr's resin mixture of 3:1 for $30 \mathrm{~min}, 1: 1$ for 1 hour, 1:3 for 1 hour then left rotating in 100\% Spurr's resin overnight. Next day samples were left in fresh Spurr's resin for approximately 6 hours then embedded over night at $65^{\circ} \mathrm{C}$. Blocks were sectioned and stained with 3\% Uranyl Acetate and Reynolds Lead citrate. Grids were examined with a Hitachi HT7700 Transmission Electron Microscope (TEM).

\section{Statistical analysis}

Muscle weights and bands from Western blots for HSPs were expressed as a ratio of the lengthened TA value to the contra-lateral control TA value. These data points were compared to values obtained from non- treated animals and a one-way ANOVA with either Dunnett's (muscle mass) or Tukey's (HSPs) post hoc testing was used to compare the fold increase in muscle mass or HSP content. The loss of peak muscle torque was determined during the $5^{\text {th }}, 10^{\text {th }}$ and last $\left(20^{\text {th }}\right)$ contraction of each set and expressed as a percentage of the first contraction. Data are reported as the mean \pm SEM. In all cases the level of significance was set at $\mathrm{p}<0.05$

\section{Results}

\section{Muscle mass}

To assess whether LCs altered muscle mass (weight wet), both contra-lateral (control) and lengthened TA muscles were removed and weighed at $0,1,3$ or 24 hours after LCs. To control for differences in muscle mass due to body size, data were compared as muscle mass relative to body mass (Table 1). In TA muscles treated with 20, 40 or 60 LCs and allowed to recover for 24 hours, a trend for increased TA muscle wet weights in the lengthened muscles was observed but a significant difference $(7.2 \% ; \mathrm{p}<0.05)$ in muscle mass was only observed after 60 LCs. When early changes $(0-3 \mathrm{hrs})$ in 
muscle mass (60 LCs) were examined, the lengthened TA mass was only significantly greater $(8.2 \%$; $\mathrm{p}<0.05$ ) than its contra-lateral (non-stimulated) control one hour after 60 LCs (Table 1) and not immediately or at three hours after LCs. Taken together, the data suggest that a single bout of LCs can elevate muscle mass.

\section{Muscle contractile torque after $\mathrm{LCs}$}

To assess the ability of each TA muscle to generate force, peak torque was measured throughout each set of LCs. Since all TA muscles were subjected to at least 1-2 sets of LCs, set data was pooled for $20(n=24)$ and $40(n=16)$ LCs. When expressed as a percentage of the first contraction, peak torque production significantly declined to approximately $74 \%(73.8 \pm 7.1 \%, \mathrm{p}<0.001)$ after 20 LCs and further declined to 44\% $(43.7 \pm 5.6 \%$; $<0.001)$ and $40 \%(39.7 \pm 5.8 \% ; \mathrm{p}<0.001)$ following 40 and $60 \mathrm{LCs}$, respectively (Figure 1). When compared to $20 \mathrm{LCs}$, the decline in torque production was significantly $(p<0.001)$ greater following 40 and 60 LCs, indicating torque loss increased in proportion to the number of LCs. When the amount of torque loss for each set of 20 LCs was examined, each set of 20 LCs resulted in significant $(\mathrm{p}<0.001)$ reductions of approximately $26 \%$ (set 1$), 32 \%$ (set 2 ) and $20 \%$ (set 3 ) in torque generating capacity per set.

\section{Intramuscular HSP content after LCs}

Hsp72 and Hsp25 content were measured in lengthened and contra-lateral (control) TA muscles 24 hours after 20, 40 or 60 LCs. Bands on Western blots were quantified and HSP content expressed as a fold increase relative to the non-contracted contra-lateral (control) TA from the same animal. In addition, a separate group of non-contracted control animals $(\mathrm{CON} ; \mathrm{n}=5)$ were used to establish baseline HSP content in the TA muscle. In general, Hsp72 content was significantly elevated in lengthened TA muscles when compared to the non-stimulated contra-lateral control TAs after 20, 40 and $60 \mathrm{LCs}$ (Figure 2). When compared to the TA from non-contracted control animals (CON), intramuscular Hsp72 content was significantly elevated after 20 LCs $(2.34 \pm 0.37$ fold, p $<0.05)$ and 
further elevated after $40(3.02 \pm 0.31$ fold, $\mathrm{p}<0.01)$ and $60 \mathrm{LCs}(3.37 \pm 0.21$ fold, $\mathrm{p}<0.001)$. The increase in Hsp72 content after 60 LCs was significantly $(\mathrm{p}<0.05)$ greater than after 20 LCs (Figure 2), suggesting the number of contractions influenced Hsp72 expression. Hsp25 content was elevated in TA muscles 24 hours after $40(3.78 \pm 0.94$ fold, $\mathrm{p}<0.01)$ and 60 LCs $(3.22 \pm 1.2$ fold, $\mathrm{p}<0.05)$, but not after 20 LCs (2.28 \pm 0.88 fold) (Figure 2). These data suggest HSP content in rat skeletal muscle increases in proportion to the number of LCs.

\section{HSF activation after LCs}

Since HSP content in the rat TA was significantly and consistently elevated 24 hours after 60 LCs, it was of interest to determine if the mechanism of HSP induction was mediated by HSF-HSE binding (termed HSF activation). To do this, HSF activation was assessed after 0, 1, 3 or 24 hours in the TA after $60 \mathrm{LC}$. HSF activation was detected in the TA muscle exposed to 60 LCs at 0,1 and 3 hours after LCs (Figure 3). There was no HSF activation observed in any of the TA muscles from contra-lateral control limbs, nor was there any HSF activation detected in TA muscles subjected to 60 LCs and allowed to recover for 24 hours. These results suggest HSF activation likely occurs rapidly and remains activated for only a few hours following the cessation of LCs.

\section{Fiber morphology after LCs}

The extent of muscle damage following 20, 40 and 60 LCs was qualitatively assessed using light microscopy. To characterize the general morphology of TA muscles subjected to LCs, muscle cross-sections were stained with Hematoxylin and Eosin. In general, non-contracted TA muscle fibers from control limbs were polygonal with a homogenous staining pattern and no evidence of inflammation or muscle damage (Figure 4 A, D, G). In the TA muscles 24 hours after 20, 40 and 60 LCs, there were a number of morphological changes indicative of muscle fiber damage when compared to non-contracted contra-lateral controls (Figure 4 B, E, H). To measure the extent of muscle damage, H\&E stained cross-sections were visually examined for leukocyte infiltration, fibre roundness and 
necrotic fibres. Table 2 shows the qualitative scores showing that TA muscles subjected to 20 lengthening contractions only showed mild damage while TA muscle subjected to either 40 or 60 LCs showed severe damage. Thus, the extent and severity of muscle damage was greater following 40 and $60 \mathrm{LCs}$ compared to $20 \mathrm{LCs}$. It is important to note that regardless of the number of LCs, the majority of muscle fibers examined appeared undamaged. However, focal regions of fiber damage in the form of necrosis and immune cell infiltration were visible in all lengthened TA muscles. After 20 LCs, damaged fibers were mostly surrounded by healthy appearing fibers while larger regions of necrosis and a greater amount of immune cell infiltration was observed in the TA after 40 and 60 LCs (Figure 5). In view of the acute reduction in muscle contractile torque, these observations further support the notion that muscle damage was greater after 40 and 60 LCs when compared to a single set of 20 LCs.

\section{Electron Microscopy}

Given that TA muscles subjected to 20 LCs showed an elevation of HSP content but relatively little visual evidence of muscle fiber damage using light microscopy, it was of interest to determine if TA muscle fibers from the 20 LC group and others showed evidence of damage at the ultra-structural level. To do this, portions of control and lengthened (20, 40 and 60 LCs) TA muscles were processed and viewed by transmission electron microscopy (Figure 6). In all sections (control and lengthened) examined, the majority of muscle fibers appeared to have undamaged Z-lines, as well as intact sarcomeres and mitochondria. However, muscles subjected to LCs showed evidence of fibers with disrupted sarcomeres and fragmented Z-lines. As the number of LCs a muscle was subjected to increased, so did the extent of disruption, such that muscles subjected to either 40 or 60 LCs exhibited greater amounts of disrupted sarcomeres and fragmented Z-lines when compared to muscles subjected to only 20 LCs. It should be noted that muscles subjected to 20 LCs showed some, albeit a lesser amount of disrupted sarcomeres and fragmented Z-lines. In all fibers subjected to LCs, no evidence of mitochondrial damage was observed. 


\section{Discussion}

In the present study, the relationship between lengthening contractions, HSP content and muscle damage was assessed in the rat TA muscle. There are several novel features of this paper. First, as few as 20 LCs increased HSP content in rat skeletal muscle. Second, the relationship between LCs and HSP content indicated that HSP content increased in proportion to the number of LCs. Last, HSF activation was detectable directly after $60 \mathrm{LCs}$, and remained elevated for up to three hours, but was not detectable at 24 hours following 60 LCs. These data suggest a low number of physiologically relevant LCs are capable of increasing skeletal muscle HSP content in an HSF mediated manner without severely compromising gross skeletal muscle architecture.

The reduction of force generating capacity is often used as an indirect marker of muscle damage and can be used to grade the severity of exercise-induced muscle damage (Paulsen et al. 2012). In the current study, muscle function as determined by peak torque, was progressively reduced following 20 , 40 or 60 LCs. As expected, multiple sets of LCs (total of 40 and 60 LCs) induced greater functional torque deficits when compared to a single set of 20 LCs. This finding is notable since greater muscle damage would result in a prolonged muscle recovery period (Paulsen et al. 2012). Although no measure of force recovery was made in the present study, the limited number of damaged fibers observed following only 20 LCs coupled with the increased number of damaged fibers observed following 40 and $60 \mathrm{LCs}$, suggests only a mild decrement in force production, if any, would be expected after 20 LCs.

It is well established that endurance exercise (Morton et al. 2006), resistance training (Paulsen et al. 2012), downhill running (Rattray et al. 2013), and isolated LCs (Holwerda and Locke 2014) all increase intramuscular HSP content. Indeed, a previous study demonstrated increased Hsp70 content in rat vastus lateralis muscles 48 hours after 90 minutes of downhill running (Rattray et al. 2013). In 
another study, Hsp70 content was elevated in the soleus of ovariectomized female rats following 90 minutes of downhill running (Bombardier et al. 2009). While prolonged downhill running appears to increase HSP content, the thermal, oxidative, metabolic stressors coupled with an excessive number of LCs during this type of exercise all may potentially contribute to any observed increase in muscle HSP content. Thus, the exact contribution of each stressor remains unclear. In the present study, there was a significant accumulation of Hsp72 in the TA 24 hours following 20, as well as after 40 or 60 LCs. Similarly, Hsp25 content was also significantly increased but only after 40 and 60 LCs. Interestingly, no differences were observed in HSP content between 40 and 60 LCs suggesting the HSP response may plateau past a set number of contractions. While all types of muscle contractions likely impose some metabolic stress, it may be the case that only LCs would impose a significant disruptive mechanical stress to a muscle fiber that results in damage and the CSR. Our previous work showing no elevation in HSP content following 100 shortening contractions but a robust elevation following 100 LCs coupled with the lack of HSPs in mice following swimming (a non-weight supported exercise) supports this possibility (Holwerda and Locke 2014; Hammond et al. 1982). Our present work suggests that LC induced stress activates the CSR elevating HSPs, perhaps in an attempt to maintain proteostasis. Our finding that Hsp72 content increased after only 20 LCs with minimal muscle damage suggests that lowvolume, minimally damaging LC protocols may be a unique method by which muscle HSP content can be elevated by exercise. Interestingly, it is well known that when a lesser number of LCs are performed prior to performing a greater number of LCs, a unique muscular protective adaptation known as "the repeated bout effect" occurs (Thompson et al. 2002). Whether an increase in HSP content following LCs contributes to the repeated bout effect and reduces the subsequent susceptibility of muscle fibers to damage when challenged by a future greater number of LC remains to be determined.

\section{HSF activation after LCs}


Intracellular HSP content is regulated by a family of heat shock factors (HSF-1 or HSF-2). In response to stress, HSF monomers dissociate from an inhibitory complex of HSPs, form trimers, translocate to the nucleus and bind to the HSE on promoter regions of the Hsp70 gene (Morimoto, 1998). In the present study, HSF activation (HSF-HSE binding) was detectable immediately after 60 LCs and remained detectable for up to 3 hours following 60 LCs. However, HSF activation was undetectable 24 hours later and in all non-contracted contra-lateral controls. This pattern of rapid, yet transient HSF activation is consistent with the response observed in striated muscle after heat shock and treadmill running (Locke and Tanguay 1996; Locke 2001; Palomero et al. 2008). Given that HSF activation was detected immediately after LCs and lasted for at least 3 hours, yet was not detected at 24 hours, it suggests the HSF activation observed was confined to the TA muscle and not the subsequent infiltrating immune cells. If the latter was responsible for the HSF activation observed, a delayed activation would be expected coinciding with the arrival of the immune cells. A rapid activation and inactivation is characteristic of HSF-1, the main stress induced regulator of HSPs. Although the exact HSF isoform detectable was not determined in the present study, the time course of activation observed suggests the HSF observed after LCs, is HSF-1. However, a limitation to our work is that we did not examine HSF activation following 20 or 40 LCs. Thus, although the observed increase in Hsp25 and Hsp72 content after 60 LCs appears to be HSF mediated, it remains possible that the elevated HSP content following 20 or 40 LC may be mediated by other factors.

\section{Relationship between LCs, muscle damage, and HSPs}

The TA muscle is comprised of fibers that vary in metabolic, contractile and anatomical characteristics (Staron et al. 1999). Although both an elevation in HSP content and muscle fiber damage was observed in TA muscles following LCs, it remains unknown if the increase in HSP content is equally elevated in each fiber throughout the entire muscle, or whether the elevated HSP expression 
is restricted to areas of localized muscle fiber damage. While this cannot be determined from the present study, the latter seems more likely since the TA muscle is primarily comprised of Fast/Type II muscle fibers and contains only a small portion of Slow/Type I fibers (Armstrong and Phelps 1984). Given that Type II fibers are more easily damaged than Type I fibers (Macaluso et al. 2012), coupled with the observations that Type I fibers are known to constitutively express higher levels of HSPs (Locke et al. 1990), it suggests that certain fibers, likely Type II, may be more easily damaged and therefore may elevate HSP expression when stressed. In addition, since LCs are also less metabolically costly than shortening contractions (Lindstedt 2016), it provides further support for the idea that exercise induced HSP increases in skeletal muscle may be more related to the cytoskeletal and sarcomeric damage than to metabolic stressors. While all contraction types likely produce a variety of stressors, such as increased temperature, $\mathrm{Ca}_{2}{ }^{+}$alterations, lowered $\mathrm{pH}$, as well as reactive oxygen species; all contributing to an overall metabolic stress, the increased mechanical strain experienced during LCs may also be a trigger for activation of the CSR and HSP upregulation. The results from the present study showing only 20 LCs were required to elevate HSP content coupled with our previous work (Holwerda and Locke, 2014) showing 100 shortening contractions did not elevate HSP content, suggests the critical signal for HSP induction may stem more from muscle damage than from metabolic perturbations. In support of this, a core temperature threshold greater than $40{ }^{\circ} \mathrm{C}$, the temperature that initiates protein denaturation, appears to be necessary to elicit a striated muscle Hsp70 response (Morton et al. 2007; Milne et al. 2011).

Although the early events following a bout of eccentric biased exercise often results in structural myofiber damage and loss of force, when LCs are performed in a controlled and progressive manner they can induce unique muscular adaptations which ultimately reduce their susceptibility to future incidents of LC-induced muscle damage - a phenomenon known as the repeated bout effect. Thus, LCs may be beneficial for athletic and clinical populations in that the adaptations to LCs 
independent of fiber damage may aid in recovery or future protection. Indeed, Friden (1984) described an apparent reorganization of sarcomeres in the absence of obvious muscle damage and necrosis after a long-term LC training program. Since this study, LC training programs have been shown to be effective at reducing the oxygen cost of exercise, treating chronic tendon injuries and causing a rightward shift in the muscles length-tension relationship (Brockett et al., 2001).

In conclusion, twenty-four hours after a single bout of $20 \mathrm{LCs}$, Hsp72 content in the TA was increased, while both Hsp72 and Hsp25 content were increased after 40 and 60 LCs. As expected, more LCs were associated with greater levels of muscle damage. The mechanism of LC-induced HSPs in the TA involves a transient increase in HSF activation (HSF-HSE binding). In the present study, as few as 20 LC to rodent muscle increased HSP content with minimal fiber necrosis and/or infiltration of immune cells. This suggests that a mild protocol of LCs (single set of 20 LCs) may also significantly increase levels of the purportedly protectively HSPs in the absence of extensive muscle damage in humans. Whether such increases can be used to benefit the recovery or rehabilitation of skeletal muscle remains to be determined.

Acknowledgement: the authors would like to thank Audrey Darabie for her assistance with the electron microscopy.

Conflict of Interest Disclaimer: the authors, E.P-T. and M.L., report no conflicts of interest associated with this manuscript. 


\section{References}

Armstrong, R.B., and Phelps R.O. 1984.Muscle fiber type composition of the rat hindlimb. Am. J. Anat. 171:259-272. doi: 10.1002/aja.1001710303 PMID: 6517030

Bombardier, E., Vigna, C., Iqbal, S., Tiidus, P.M., and Tupling, A.R. 2009. Effects of ovarian sex hormones and downhill running on fiber-type-specific HSP70 expression in rat soleus. J. Appl. Physiol. 106:2009-2015. doi: 10.1152/japplphysiol.91573.2008PMID: 19359608

Brockett C.L., Morgan, D.L., Proske, U. 2001. Human hamstring muscles adapt to eccentric exercise by changing optimum length. Med. Sci. Sports Exerc. 33:783-90. PMID: 11323549

Frier, B.C, and Locke M. 2007. Heat stress inhibits skeletal muscle hypertrophy. Cell Stress Chaperones.12:132-141. PMID: 17688192

Hammond, G.L., Lai, Y.K., and Markert, C.L. 1982. Diverse forms of stress lead to a new pattern of gene expression through a common and essential metabolic pathway. Proc. Natl. Acad. Sci. USA.79:3485-3488. PMID: 6954493

Holwerda, A.M., and Locke, M. 2014. Hsp25 and Hsp72 content in rat skeletal muscle following controlled shortening and lengthening contractions. Appl. Physiol. Nutr. Metab. 39:1380-1387. doi: 10.1139/apnm-2014-0118 PMID: 25356915

Laemmli, U. 1970. Cleavage of structural proteins during the assembly of the head of bacteriophage 
T4. Nature. 227:680-685. PMID: 5432063

Lindstedt, S.L. 2016. Skeletal muscle tissue in movement and health: positives and negatives. J. Exp. Biol. 219:183-188. doi: 10.1242/jeb.124297 PMID: 26792329

Locke, M., Noble, E.G., and Atkinson, B.G. 1991. Inducible isoform of HSP70 is constitutively expressed in a muscle fiber type specific pattern. Am. J. Physiol. 261:774-779. PMID: 1951668

Locke, M., and Tanguay, R.M. 1996. Increased HSF activation in muscles with a high constitutive Hsp70 expression. Cell Stress Chaperones.1:189-196. PMID: 9222604

Locke, M. 2001. Heat shock transcription factor activation and hsp72 accumulation in aged skeletal muscle. Cell Stress Chaperones.5:45-51. PMID: 10701839

Locke, M. 2008. Heat shock protein accumulation and heat shock transcription factor activation in rat skeletal muscle during compensatory hypertrophy. Acta. Physiol. 192:403-411. doi: 10.1111/j.17481716.2007.01764.xPMID: 17973955

Lowry, O.H., Rosebrough, N.J., Farr, A.L., and Randall, R.J. 1951. Protein measurement with the folin phenol reagent. J. Biol. Chem.193:265-275.PMID: 14907713

Macaluso, F., Isaacs, A.W., and Myburgh, K.H. 2012. Preferential type II muscle fiber damage from plyometric exercise. J. Athl. Train. 47:414-420. doi: 10.4085/1062-6050-47.4.13

PMID: 22889657 
McCully, K.K., and Faulkner, J.A. 1985. Injury to skeletal muscle fibers of mice following lengthening contractions. J. Anat. 59:119-126. PMID: 4030553

Milne, K.J., Thorp, D.B., Krause, M., and Noble, E.G. 2011. Core temperature is a greater influence than endogenous $17 \beta$-estradiol on the exercise-induced accumulation of myocardial heat shock protein mRNA. Can. J. Physiol. Pharmacol. 89:855-860. doi: 10.1139/y11-089 PMID: 22039956

Morimoto, R.I. 1998. Regulation of the heat shock transcriptional response: cross talk between a family of heat shock factors, molecular chaperones, and negative regulators. Genes Dev.12:3788-3796. PMID: 9869631

Morton, J.P., Maclaren, D.P., Cable, N.T., Campbell, I.T., Evans, L., and Bongers, T. et al. 2007. Elevated core and muscle temperature to levels comparable to exercise do not increase heat shock protein content of skeletal muscle of physically active men. Acta. Physiol. 190:319-327. doi: PMID:

Morton, J.P., McLaren, D.P.M., Cable, N.T., Bongers, T., Griffiths, R.D., and Campbell, I.T., et al. 2006. Time course and differential responses of the major heat shock protein families in nhuman skeletal muscle following acute nondamaging treadmill exercise. J. Appl. Physiol. 101:176-182. doi: 10.1111/j.1748-1716.2007.01711.x PMID: 17488245

Palomero, J., Broome, C.S., Rasmussen, P., Mohr., M., Nielsen, B., and Nybo, L., et al. 2008. Heat shock factor activation in human muscles following a demanding intermittent exercise protocol is 
attenuated with hyperthermia. Acta. Physiol. 193:79-88. doi: 10.1111/j.1748-1716.2007.01774.x PMID: 18081888

Paulsen, G., Hanssen, K.E., Ronnestad, B.R., Kvamme, N.H., Ugelstad, I., and Kadi, F., et al. 2012. Strength training elevates HSP27, HSP70 and $\alpha \beta$-crystallin levels in musculi vastus lateralis and trapezius. Eur. J. Appl. Physiol.112:1773-1782. doi: 10.1007/s00421-011-2132-8 PMID: 21901266

Paulsen, G., Mikkelsen, U.R., Raastad, T., and Peake, J.M. 2012. Leucocytes, cytokines and satellite cells: what role do they play in muscle damage and regeneration following eccentric exercise? Exerc. Immunol. Rev.18:42-97. PMID: 22876722

Paulsen, G., Vissing, K., Kalhovde, J.M., Ugelstad, I., Bayer, M.L., and Kadi, F., et al. 2007. Maximal eccentric exercise induces a rapid accumulation of small heat shock proteins on myofibrils and a delayed HSP70 response in humans. Am. J. Physiol. Regul. Integr. Comp. Physiol.293:844-853. doi: 10.1152/ajpregu.00677.2006 PMID: 17522120

Pirkkala, L., Nykänen, P., and Sistonen, L. 2001. Roles of the heat shock transcription factors in regulation of the heat shock response and beyond. FASEB. J.15:1118-131. PMID: 11344080

Proske, U., and Morgan, D.L. 2001. Muscle damage from eccentric exercise: mechanism, mechanical signs, adaptation and clinical applications. J. Physiol. 537(Pt 2):333-345. PMID: 11731568 
Rattray, B., Thompson, M., Ruell, P., and Caillaud, C. 2013. Specific training improves skeletal muscle mitochondrial calcium homeostasis after eccentric exercise. Eur. J. Appl. Physiol.113:427-436. doi: 10.1007/s00421-012-2446-1 PMID: 22777498

Senf, S.M. 2013. Skeletal muscle heat shock protein 70: diverse functions and therapeutic potential for wasting disorders. Front. Physiol. 4: 1-6. doi: 10.3389/fphys.2013.00330PMID: 24273516

Staron, R.S., Kraemer, W.J., Hikida, R.S., Fry, A.C., Murray, J.D., and Campos, G.E. 1999. Fiber type composition of four hindlimb muscles of adult Fisher 344 rats. Histochem. Cell. Biol.111:117-123. doi: PMID:

Thompson, H.S., Clarkson, P.M., and Scordilis, S.P. 2002. The repeated bout effect and heat shock proteins: intramuscular HSP27 and HSP70 expression following two bouts of eccentric exercise in humans. Acta. Physiol. Scand. 174:47-56. PMID: 10090572

Touchberry, C.D., Gupte, A.A., Bomhoff, G.L., Graham, Z.A., Geiger, P.C., and Gallagher, P.M. 2012. Acute heat stress prior to downhill running may enhance skeletal muscle remodeling. Cell Stress Chaperones.17:693-705. doi: 10.1007/s12192-012-0343-5 PMID: 22589083

Towbin, H., Staehelin, T., and Gordon, J. 1979. Electrophoretic transfer of proteins from polyacrylamide gels to nitrocellulose sheets: procedure and some applications. Proc. Natl. Acad. Sci. USA.76:4350-4354. PMID: 388439 
Table 1: Changes in muscle mass after LCs.

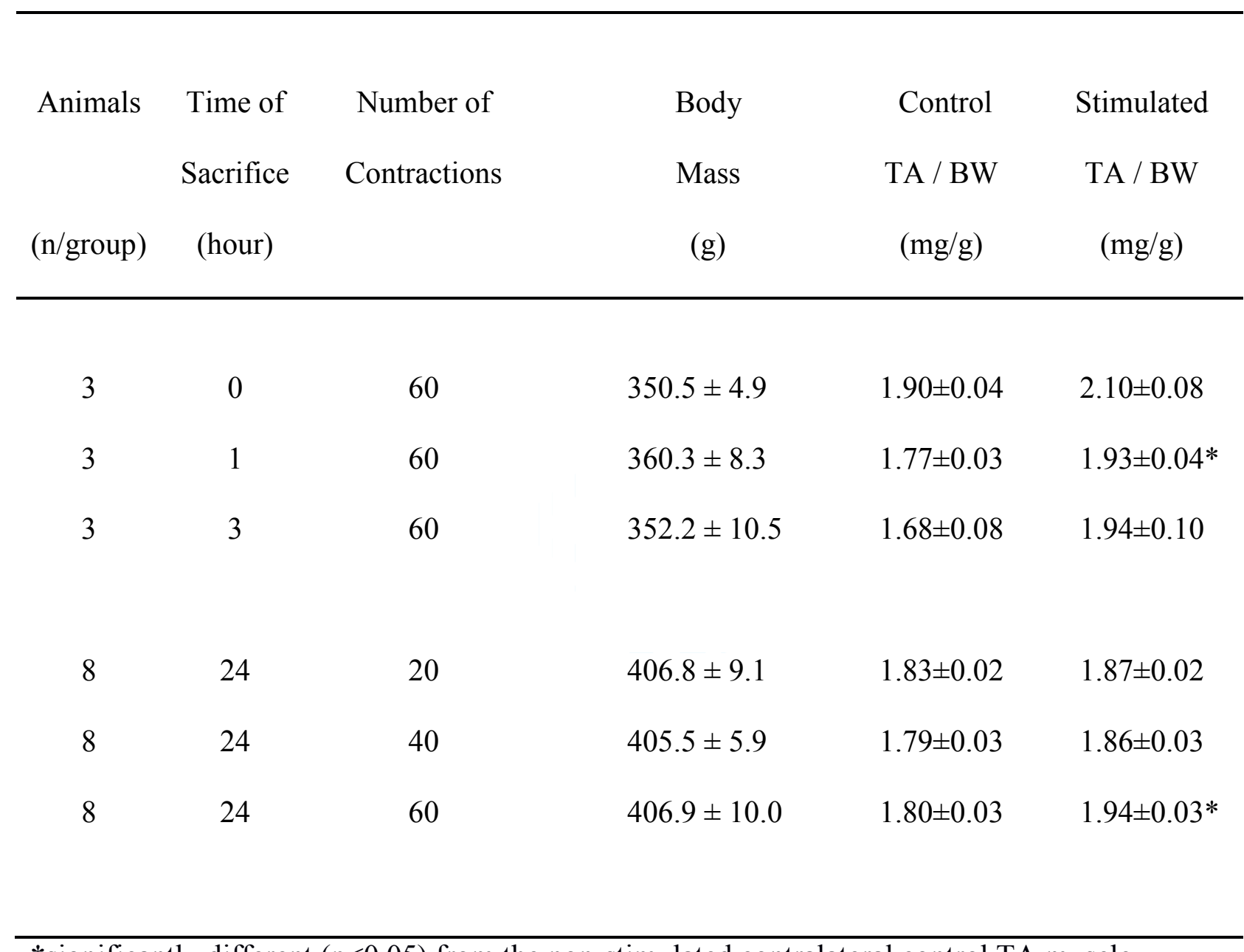

\footnotetext{
*significantly different $(\mathrm{p}<0.05)$ from the non-stimulated contralateral control TA muscle.
} 
Table 2: Qualitative assessment of muscle damage after 20, 40 and 60 LCs.

\begin{tabular}{cccc}
\hline & Leukocytes & Rounded fibers & Necrotic fibers \\
\hline 20 LCs & + & + & + \\
40 LCs & +++ & +++ & +++ \\
60 LCs & +++ & +++ & +++ \\
& & & \\
\hline$:+$ denotes mild,+++ denotes moderate to severe.
\end{tabular}

$:+$ denotes mild, +++ denotes moderate to severe. 


\section{Figure Legends}

Figure 1: Peak muscle torque $(\mathrm{g}-\mathrm{cm})$ immediately following 20, 40 and 60 LCs. Data are normalized to muscle mass and presented as a percentage of the first contraction. *denotes significant $(\mathrm{p}<0.001)$ difference from the $1^{\text {st }}$ LC. Data are presented as the mean \pm SEM and the level of significance was set at $\mathrm{p}<0.05$ ( $\mathrm{n}=24,16$ and 8 per set).

Figure 2: Hsp72 and Hsp25 content increases in the TA muscle 24 hours after LCs. Panel A. Representative immunoblots for Hsp72 and Hsp25 in control animals (CON), as well as after 20, 40 and 60 LCs. Lane 1: CON, lane 2: 20 LCs, lane 3: 40 LCs, lane 4: 60 LCs. Panel B. Band densitometry expressed relative to the non-stimulated control limbs. *denotes significant $(p<0.05)$ difference from CON rats. $\uparrow$ denotes significant $(p<0.05)$ difference from 20 LCs. Data are presented as the mean \pm SEM and the level of significance was set at $\mathrm{p}<0.05$ ( $\mathrm{n}=7$ /group).

Figure 3: HSF activation in the TA after 60 LCs. Panel A: Representative EMSA after 60 LCs. Lane 1: control TA immediately (0 hr) after 60 LCs. Lane 2: stimulated TA immediately after 60 LCs. Lane 3: control TA 1 hr. Lane 4: stimulated TA 1 hr. Lane 5: control TA 3 hr. Lane 6: stimulated TA $3 \mathrm{hr}$. Lane 7: control TA $24 \mathrm{hr}$. Lane 8: stimulated TA $24 \mathrm{hr}$. 9: positive control (pure HSF). Panel B: Close-up of area of interest (HSF band). NS: non-specific binding, CB: constitutive binding, HSF: heat shock transcription factor.

Figure 4: Muscle fiber damage 24 hours after 20, 40 and 60 LCs. Panels A, D, G: Controls. Panels B, E, H: Localized (focal) muscle fiber damage after 20, 40 and 60 LCs. Panels C, F, I: Insets showing necrotic fibers from panels B, E, H. Scale bars $=100 \mu \mathrm{m}$. Inset scale bars $=25 \mu \mathrm{m}$. 
Figure 5: Regions of muscle fiber damage 24 hours after 20, 40 and 60 LCs. When compared to control TA samples (Panel A), necrotic fibers were visible in all stimulated TA samples after 20 (Panel B), 40 (Panels C, D) and 60 LCs (Panels E, F); however, the extent of necrosis was greatest after 40 or 60 LCs. Arrows indicate regions of fiber necrosis. Scale bars $=100 \mu \mathrm{m}$

Figure 6: Ultrastructural damage 24 hours after LCs. Muscle samples were viewed using TEM. Panel A: Control TA. Panel B: TA after 20 LCs. Panel C: TA after 40 LC. Panel D: TA after 60 LCs. Scale bars $=2.0 \mu \mathrm{m}$. 


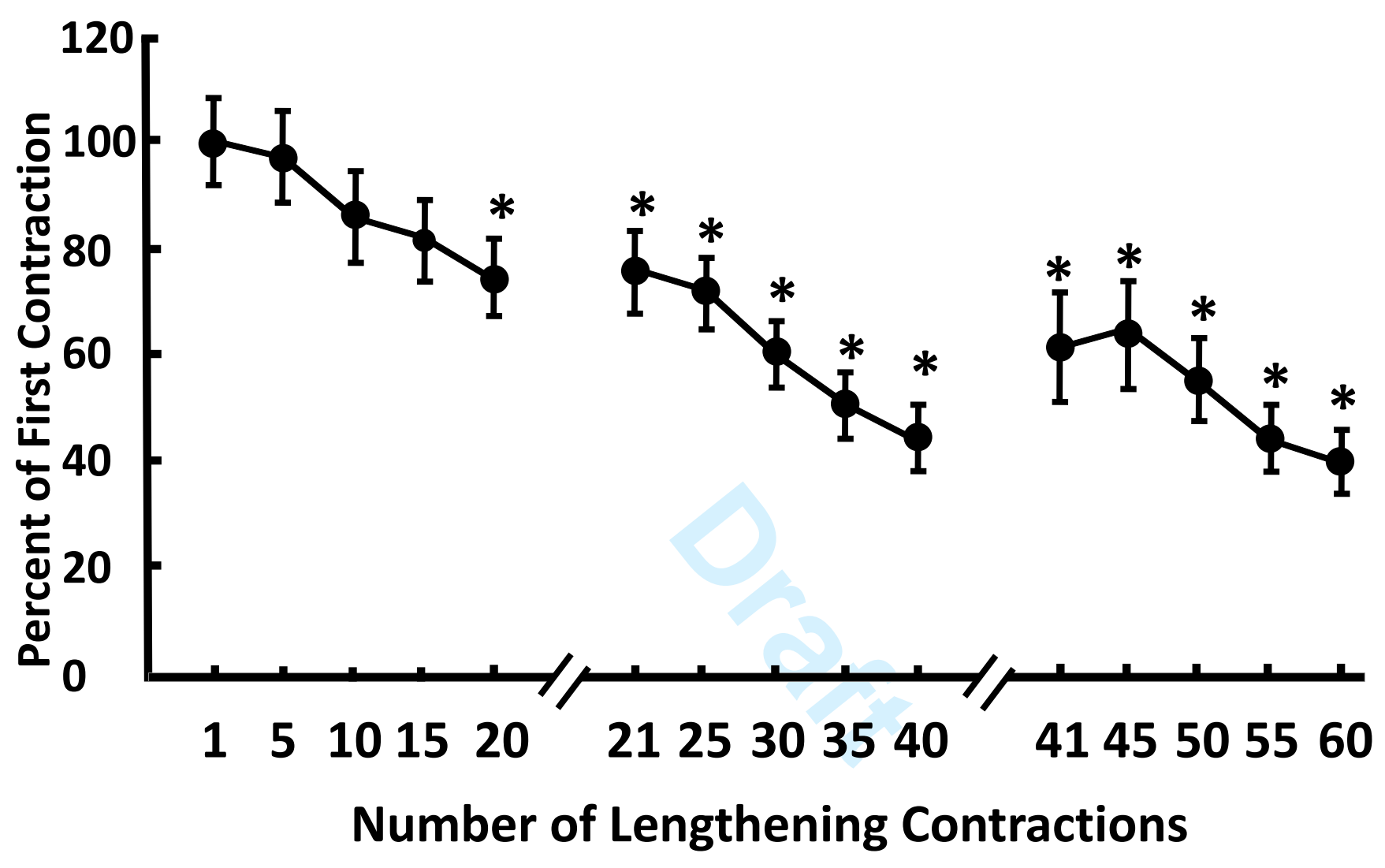


A.

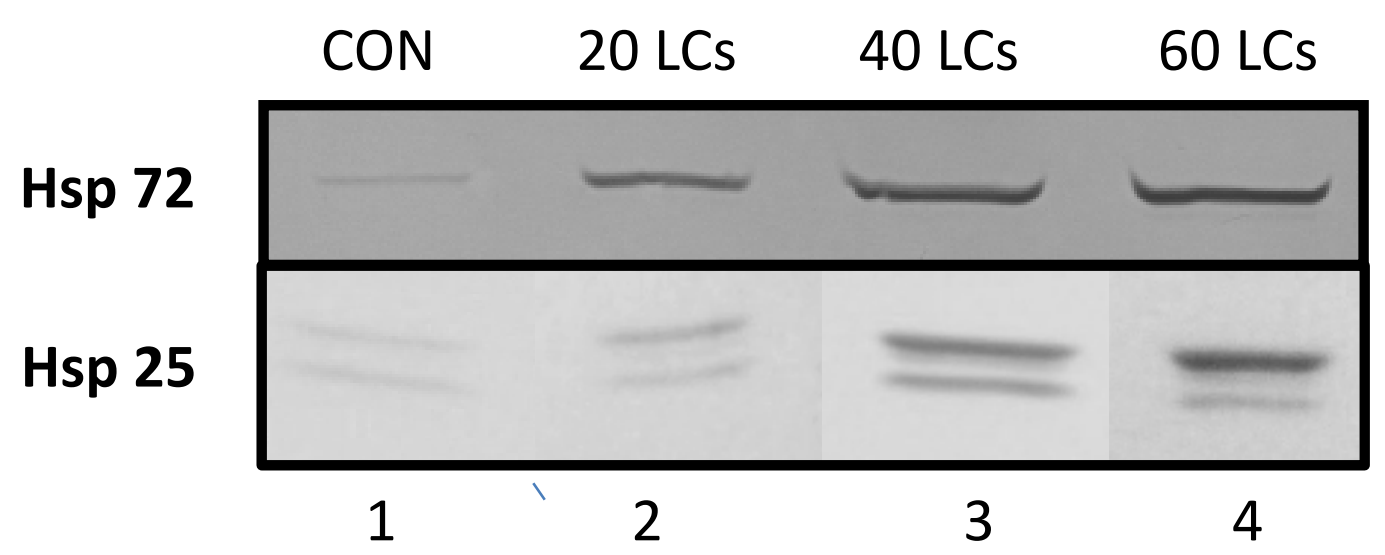

B.

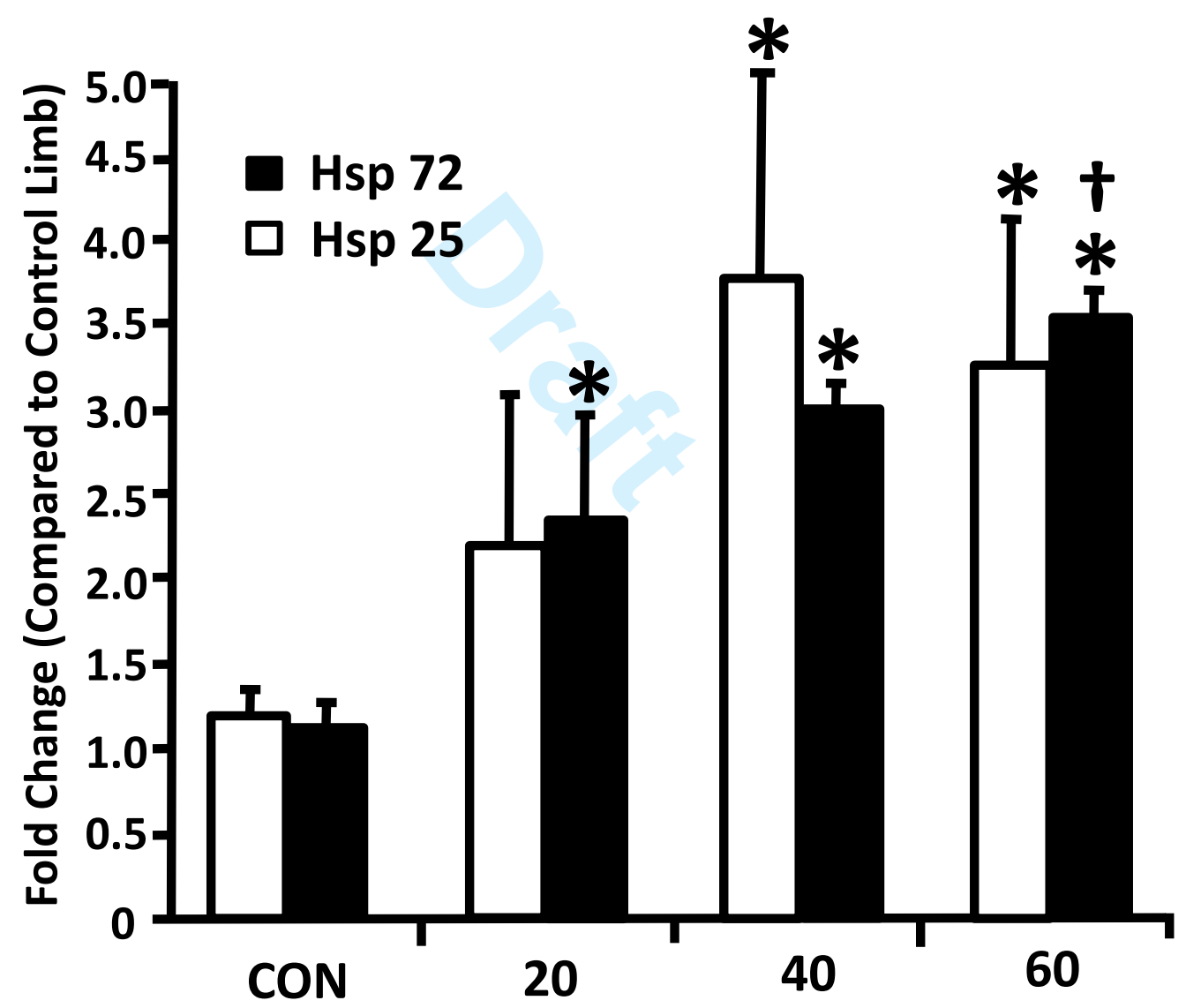


pure

A

C $\quad 0 \mathrm{~h} \quad \mathrm{C} \quad 1 \mathrm{~h} \quad \mathrm{C} \quad 3 \mathrm{~h} \quad \mathrm{C} \quad 24 \mathrm{~h} \quad \mathrm{HSF}$

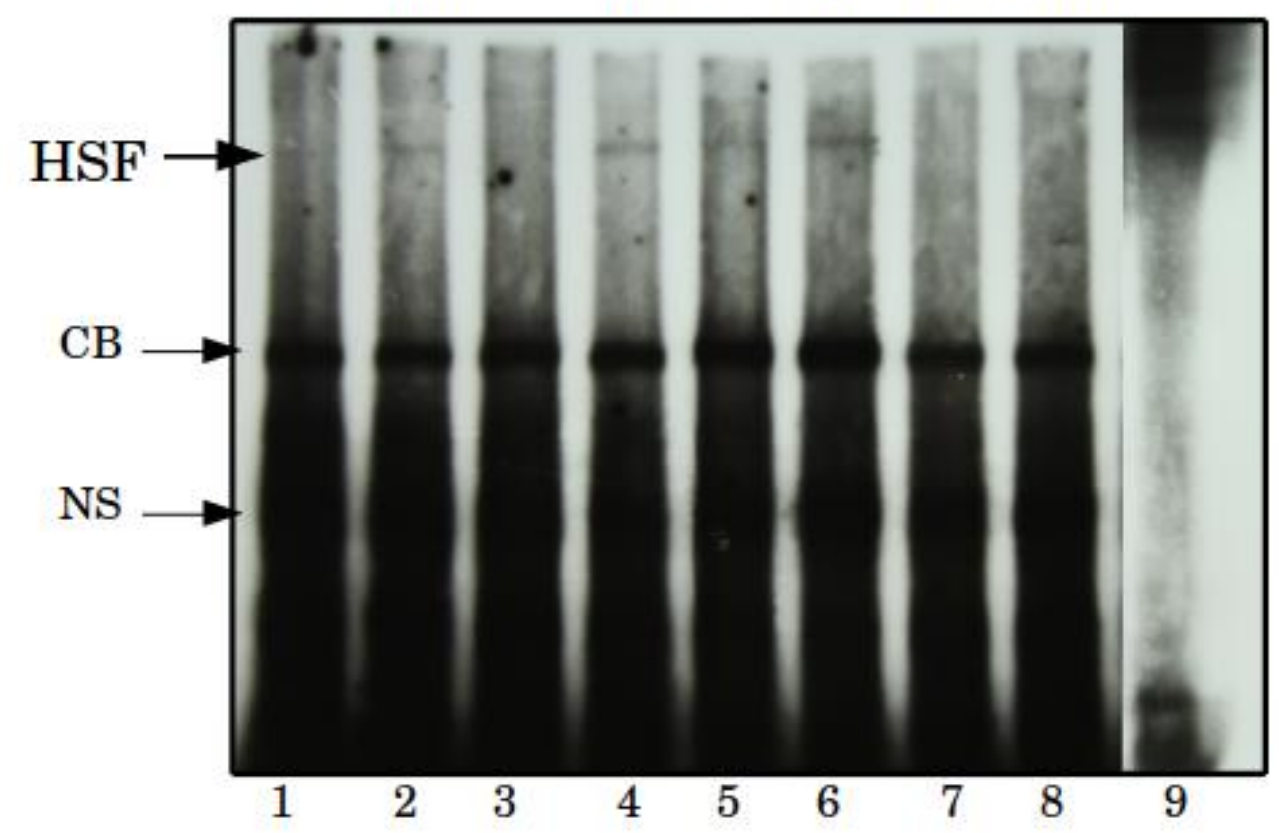

B \begin{tabular}{cccccccccc}
$\mathrm{C}$ & $0 \mathrm{~h}$ & $\mathrm{C}$ & $1 \mathrm{~h}$ & $\mathrm{C}$ & $3 \mathrm{~h}$ & $\mathrm{C}$ & $24 \mathrm{hr}$ & $\mathrm{HSF}$ \\
\hline & & & & & & & & & \\
\hline
\end{tabular} 


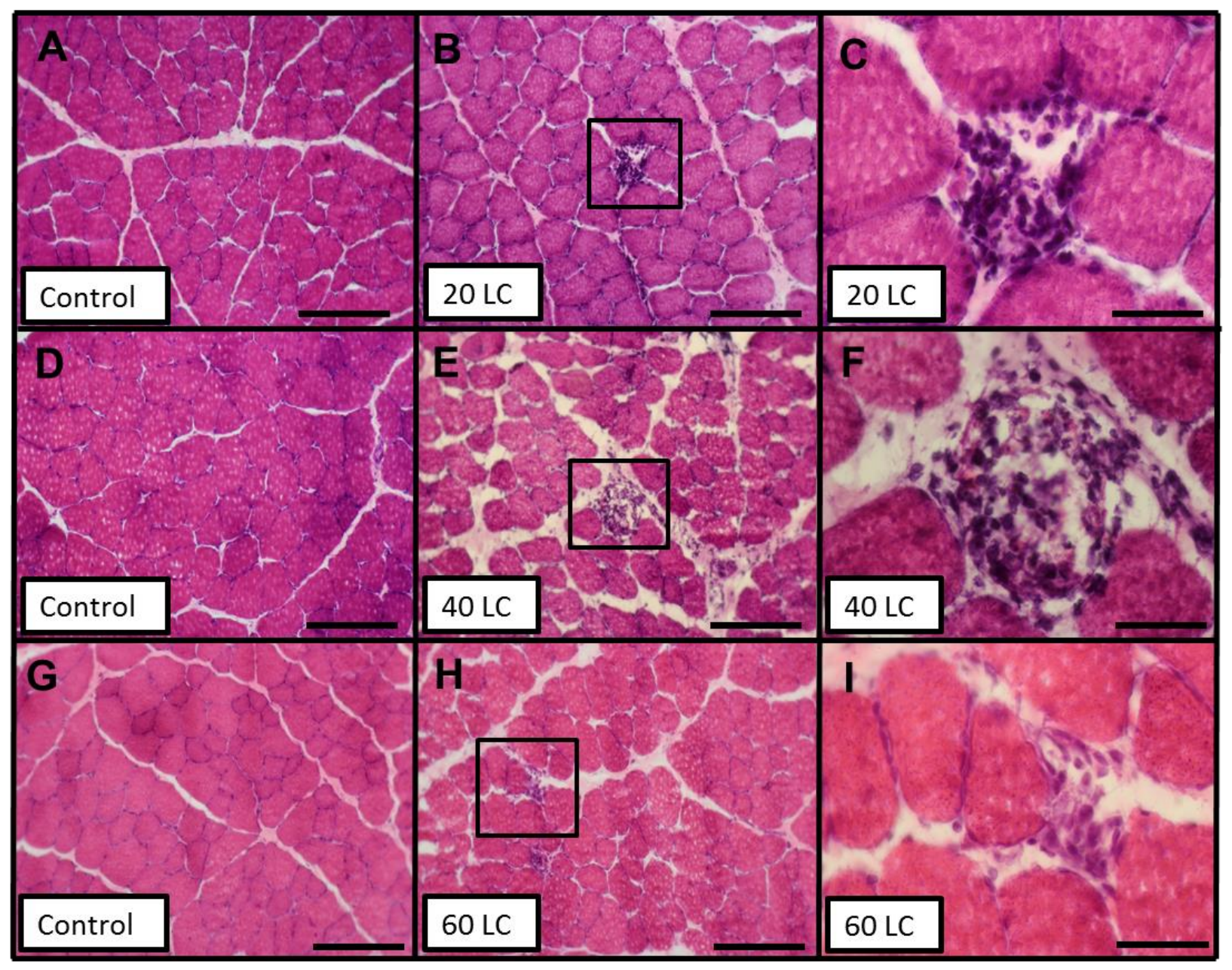




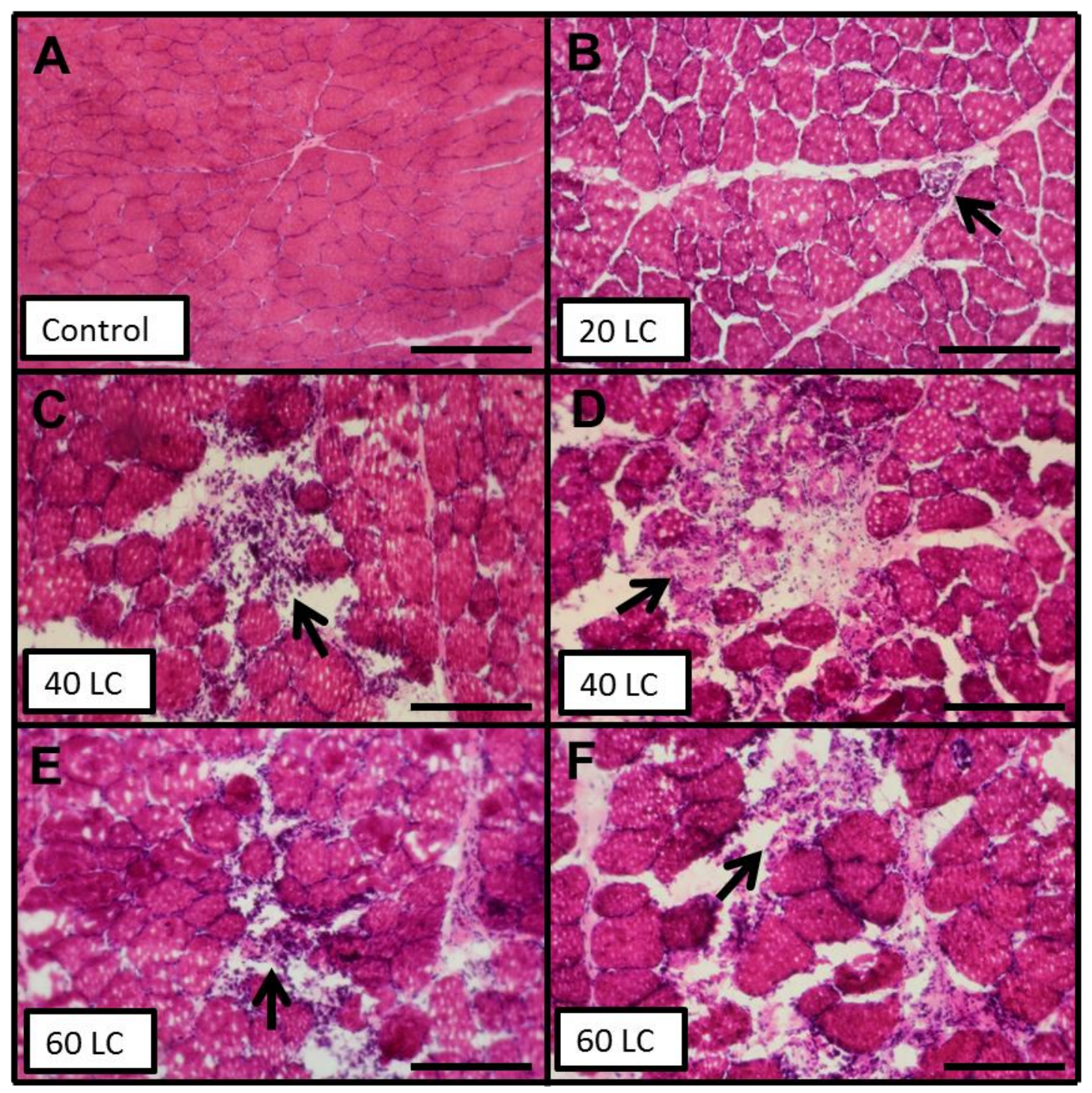




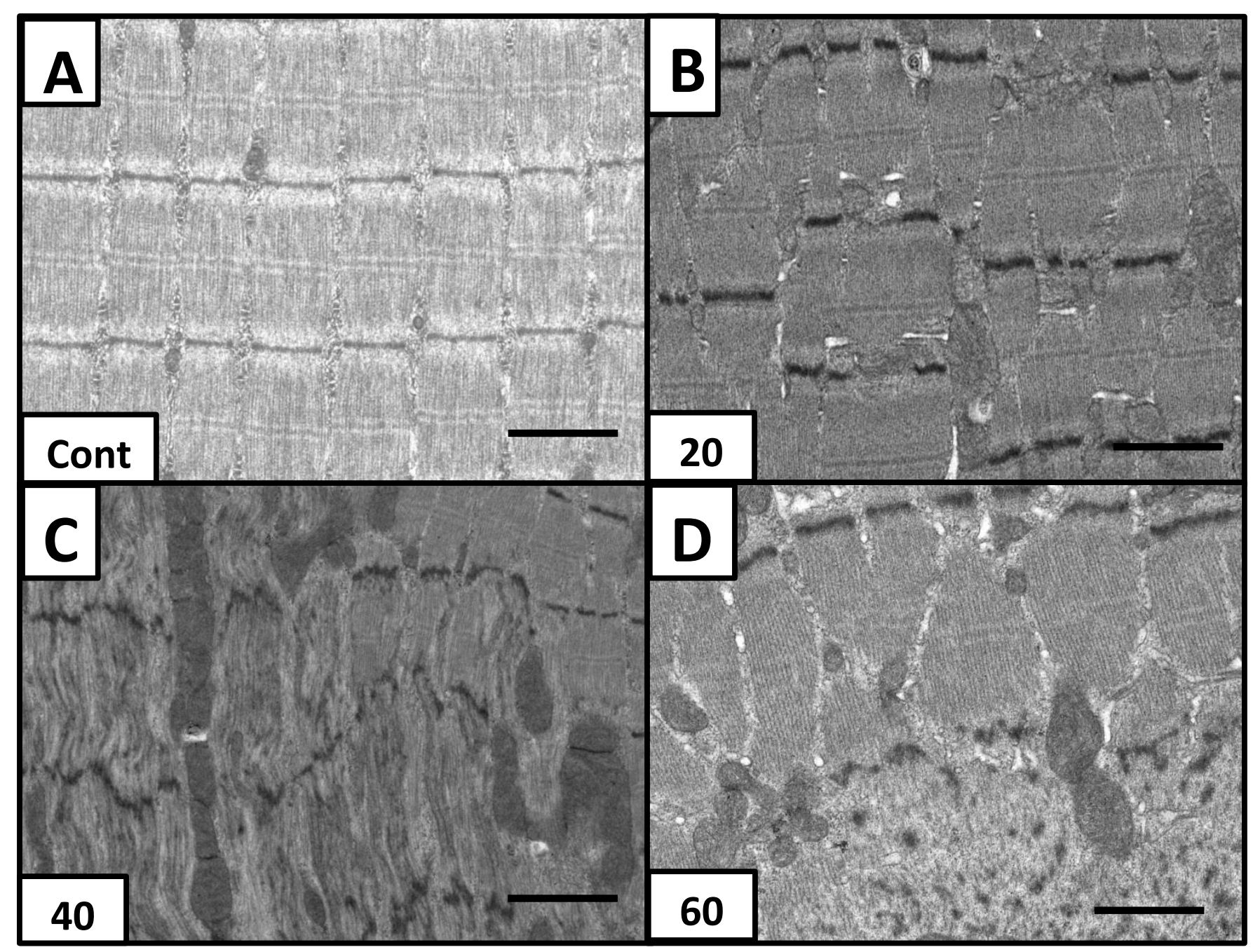

